\title{
\begin{tabular}{l|l} 
Mibraries & DSpace@MIT
\end{tabular}
}

\author{
MIT Open Access Articles
}

\section{DISCOVERY AND COSMOLOGICAL IMPLICATIONS OF SPT-CL J2106-5844, THE MOST MASSIVE KNOWN CLUSTER AT z > 1}

The MIT Faculty has made this article openly available. Please share how this access benefits you. Your story matters.

Citation: Foley, R. J., K. Andersson, G. Bazin, T. de Haan, J. Ruel, P. A. R. Ade, K. A. Aird, et al. “DISCOVERY AND COSMOLOGICAL IMPLICATIONS OF SPT-CL J2106-5844, THE MOST MASSIVE KNOWN CLUSTER AT z >1." The Astrophysical Journal 731, no. 2 (March 28, 2011): 86. (C) 2011 The American Astronomical Society

As Published: http://dx.doi.org/10.1088/0004-637x/731/2/86

Publisher: IOP Publishing

Persistent URL: http://hdl.handle.net/1721.1/96775

Version: Final published version: final published article, as it appeared in a journal, conference proceedings, or other formally published context

Terms of Use: Article is made available in accordance with the publisher's policy and may be subject to US copyright law. Please refer to the publisher's site for terms of use. 


\title{
DISCOVERY AND COSMOLOGICAL IMPLICATIONS OF SPT-CL J2106-5844, THE MOST MASSIVE KNOWN CLUSTER AT $z>1$
}

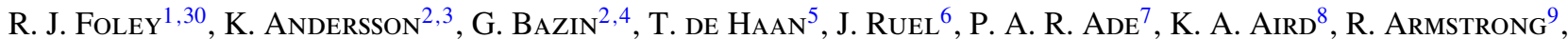

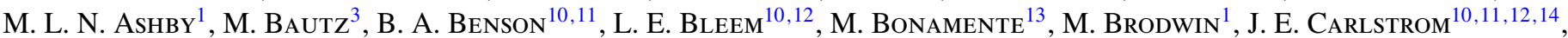 \\ C. L. Chang ${ }^{10,11}$, A. Clocchiatti ${ }^{15}$, T. M. Crawford ${ }^{10,14}$, A. T. Crites ${ }^{10,14}$, S. Desai ${ }^{9,16}$, M. A. Dobbs ${ }^{5}$, J. P. Dudley ${ }^{5}$,
} G. G. Fazio ${ }^{1}$, W. R. Forman ${ }^{1}$, G. Garmire ${ }^{17}$, E. M. George ${ }^{18}$, M. D. Gladders ${ }^{10,14}$, A. H. GonZalez ${ }^{19}$, N. W. Halverson ${ }^{20}$, F. W. High ${ }^{10,14}$, G. P. Holder ${ }^{5}$, W. L. HolzaPfel ${ }^{18}$, S. Hoover ${ }^{10,11}$, J. D. Hrubes ${ }^{8}$, C. Jones ${ }^{1}$, M. Joy ${ }^{21}$, R. KeISLER ${ }^{10,12}$, L. KNOX ${ }^{22}$, A. T. LeE $^{18,23}$, E. M. Leitch ${ }^{10,14}$, M. LuekeR ${ }^{18}$, D. Luong-VAN ${ }^{8}$, D. P. MARrone ${ }^{10,8}$, J. J. McMahoN ${ }^{10,11,24}$, J. Mehl ${ }^{10,14}$, S. S. Meyer ${ }^{10,11,12,14}$, J. J. Mohr ${ }^{2,4,25}$, T. E. Montroy ${ }^{26}$, S. S. MurraY ${ }^{1}$, S. Padin ${ }^{10,14,27}$, T. PlagGE ${ }^{10,14}$, C. Pryke ${ }^{10,11,14}$, C. L. Reichardt ${ }^{18}$, A. Rest ${ }^{6,28}$, J. E. Ruhl ${ }^{26}$, B. R. SaliwanchiK ${ }^{26}$, A. Saro ${ }^{2}$, K. K. Schaffer ${ }^{10,11}$, L. Shaw ${ }^{5,29}$, E. Shirokofr ${ }^{18}$, J. Song ${ }^{24}$, H. G. Spieler ${ }^{23}$, B. Stalder ${ }^{1}$, S. A. Stanford ${ }^{22}$, Z. Staniszewski ${ }^{26}$, A. A. Stark ${ }^{1}$,

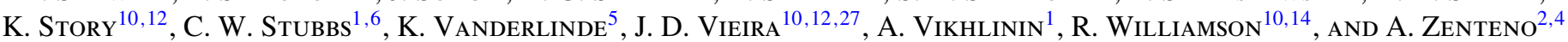

${ }^{1}$ Harvard-Smithsonian Center for Astrophysics, 60 Garden Street, Cambridge, MA 02138, USA; rfoley@ cfa.harvard.edu

${ }^{2}$ Department of Physics, Ludwig-Maximilians-Universität, Scheinerstr. 1, 81679 München, Germany

${ }^{3}$ MIT Kavli Institute for Astrophysics and Space Research, Massachusetts Institute of Technology, 77 Massachusetts Avenue, Cambridge, MA 02139, USA

${ }^{4}$ Excellence Cluster Universe, Boltzmannstr. 2, 85748 Garching, Germany

${ }^{5}$ Department of Physics, McGill University, 3600 Rue University, Montreal, Quebec H3A 2T8, Canada

${ }^{6}$ Department of Physics, Harvard University, 17 Oxford Street, Cambridge, MA 02138, USA

${ }^{7}$ Department of Physics and Astronomy, Cardiff University, CF24 3YB, UK

${ }^{8}$ University of Chicago, 5640 South Ellis Avenue, Chicago, IL 60637, USA

${ }^{9}$ National Center for Supercomputing Applications, University of Illinois, 1205 West Clark Street, Urbanan, IL 61801, USA

${ }^{10}$ Kavli Institute for Cosmological Physics, University of Chicago, 5640 South Ellis Avenue, Chicago, IL 60637, USA

${ }^{11}$ Enrico Fermi Institute, University of Chicago, 5640 South Ellis Avenue, Chicago, IL 60637, USA

${ }^{12}$ Department of Physics, University of Chicago, 5640 South Ellis Avenue, Chicago, IL 60637, USA

${ }^{13}$ Physics Department, University of Alabama in Huntsville, Huntsville, AL 35899, USA

${ }^{14}$ Department of Astronomy and Astrophysics, University of Chicago, 5640 South Ellis Avenue, Chicago, IL 60637, USA

${ }^{15}$ Departamento de Astronomia y Astrofisica, Pontificia Universidad Catolica, Casilla 306, Santiago 22, Chile

${ }^{16}$ Department of Astronomy, University of Illinois, 1002 West Green Street, Urbana, IL 61801, USA

${ }^{17}$ Department of Astronomy and Astrophysics, Pennsylvania State University, 525 Davey Lab, University Park, PA 16802, USA

${ }^{18}$ Department of Physics, University of California, Berkeley, CA 94720, USA

${ }^{19}$ Department of Astronomy, University of Florida, Gainesville, FL 32611, USA

${ }^{20}$ Department of Astrophysical and Planetary Sciences and Department of Physics, University of Colorado, Boulder, CO 80309, USA

${ }^{21}$ Department of Space Science, VP62, NASA Marshall Space Flight Center, Huntsville, AL 35812, USA

22 Department of Physics, University of California, One Shields Avenue, Davis, CA 95616, USA

${ }^{23}$ Physics Division, Lawrence Berkeley National Laboratory, Berkeley, CA 94720, USA

${ }^{24}$ Department of Physics, University of Michigan, 450 Church Street, Ann Arbor, MI, 48109, USA

${ }^{25}$ Max-Planck-Institut für extraterrestrische Physik, Giessenbachstr. 85748 Garching, Germany

${ }^{26}$ Physics Department, Case Western Reserve University, Cleveland, OH 44106, USA

${ }^{27}$ California Institute of Technology, 1200 E. California Blvd., Pasadena, CA 91125, USA

${ }^{28}$ Space Telescope Science Institute, 3700 San Martin Dr., Baltimore, MD 21218, USA

${ }^{29}$ Department of Physics, Yale University, P.O. Box 208210, New Haven, CT 06520-8120, USA Received 2011 January 6; accepted 2011 February 4; published 2011 March 28

\begin{abstract}
Using the South Pole Telescope (SPT), we have discovered the most massive known galaxy cluster at $z>1$, SPT-CL J2106-5844. In addition to producing a strong Sunyaev-Zel'dovich (SZ) effect signal, this system is a luminous X-ray source and its numerous constituent galaxies display spatial and color clustering, all indicating the presence of a massive galaxy cluster. Very Large Telescope and Magellan spectroscopy of 18 member galaxies shows that the cluster is at $z=1.132_{-0.003}^{+0.002}$. Chandra observations obtained through a combined HRC-ACIS GTO program reveal an X-ray spectrum with an Fe K line redshifted by $z=1.18 \pm 0.03$. These redshifts are consistent with the galaxy colors found in optical, near-infrared, and mid-infrared imaging. SPT-CL J2106-5844 displays extreme X-ray properties for a cluster having a core-excluded temperature of $T_{X}=11.0_{-1.9}^{+2.6} \mathrm{keV}$ and a luminosity (within $\left.r_{500}\right)$ of $L_{X}(0.5-2.0 \mathrm{keV})=(13.9 \pm 1.0) \times 10^{44} \mathrm{erg} \mathrm{s}^{-1}$. The combined mass estimate from measurements of the SZ effect and X-ray data is $M_{200}=(1.27 \pm 0.21) \times 10^{15} h_{70}^{-1} M_{\odot}$. The discovery of such a massive gravitationally collapsed system at high redshift provides an interesting laboratory for galaxy formation and evolution, and is a probe of extreme perturbations of the primordial matter density field. We discuss the latter, determining that, under the assumption of $\Lambda \mathrm{CDM}$ cosmology with only Gaussian perturbations, there is only a $7 \%$ chance of finding a galaxy cluster similar to SPT-CL J2106-5844 in the $2500 \mathrm{deg}^{2}$ SPT survey region and that only one such galaxy cluster is expected in the entire sky.
\end{abstract}

Key words: early universe - galaxies: clusters: individual (SPT-CL J2106-5844) - galaxies: evolution - galaxies: formation - large-scale structure of universe

Online-only material: color figures 


\section{INTRODUCTION}

Galaxy clusters are the most massive collapsed objects in the universe. Massive clusters form from the most overdense perturbations on their mass scale in the primordial matter density field and are exceedingly rare objects. At high redshift, corresponding to a relatively short time after the big bang, they provide a powerful probe of the initial matter density field of the universe. Their abundance is a particularly sensitive probe of Gaussianity in the matter density field (e.g., Lucchin \& Matarrese 1988; Colafrancesco et al. 1989; Mortonson et al. 2011) and, as a result, cluster surveys can test various models of inflation and topological defects (e.g., Verde et al. 2001). Observations of galaxy clusters, in which the constituent galaxies have similar formation histories, can strongly constrain hierarchical formation models (e.g., De Lucia \& Blaizot 2007); high-mass clusters at high redshift are particularly interesting astronomical laboratories for galaxy formation and evolution.

The bulk of baryonic matter in galaxy clusters is in the form of fully ionized intracluster gas. The free electrons in this gas emit thermal Bremsstrahlung radiation in the X-ray and lead to inverse Compton scattering of the cosmic microwave background (CMB) photons. This scattering results in a spectral distortion of the CMB observed toward the cluster known as the thermal Sunyaev-Zel'dovich effect (SZ; Sunyaev \& Zeldovich 1972). The surface brightness of the SZ effect is determined solely by the thermal pressure of the intracluster medium integrated along the line of sight and is independent of the cluster redshift. The total SZ signal of a cluster is a measure of its total thermal energy and is therefore tightly correlated with the cluster mass. This makes the SZ effect a powerful technique for discovering galaxy clusters at high redshift and an efficient means of finding the highest-mass clusters (e.g., Carlstrom et al. 2002).

With the goal of detecting galaxy clusters using the SZ effect, the $10 \mathrm{~m}$ South Pole Telescope (SPT; Carlstrom et al. 2009) is surveying $2500 \mathrm{deg}^{2}$ in three bands at millimeter wavelengths. The SPT survey is sensitive to galaxy clusters with a mass $\gtrsim 5 \times 10^{14} M_{\odot}$ at all redshifts. The first three SZ-discovered clusters were reported by the SPT collaboration (Staniszewski et al. 2009). The SPT collaboration recently published the full $5 \sigma$ cluster catalog from the first $200 \mathrm{deg}^{2}$ of the survey, observed during the 2008 observing season (Vanderlinde et al. 2010). Most recently, a sample of the 26 most massive galaxy clusters extracted from the full $2500 \mathrm{deg}^{2}$ SPT survey has been used to test the standard cosmological model (Williamson et al. 2011). We refer the reader to these previous SPT papers for a complete description of the survey strategy and goals. The Atacama Cosmology Telescope (ACT) has also recently reported their initial catalog of SZ-discovered clusters (Marriage et al. 2010).

The discovery of $z \gtrsim 1$ galaxy clusters has been challenging. Recently, several groups have detected galaxy clusters at $z \gtrsim 1.1$, with most either being found through X-ray (e.g., Rosati et al. 2004; Mullis et al. 2005; Stanford et al. 2006; Rosati et al. 2009) or Spitzer mid-infrared (MIR) imaging (e.g., Stanford et al. 2005; Brodwin et al. 2006; Eisenhardt et al. 2008; Muzzin et al. 2009; Wilson et al. 2009; Papovich et al. 2010). Only one SZ-detected discovered galaxy cluster has been spectroscopically confirmed at $z>1$ prior to the results presented here (Brodwin et al. 2010). In principle, high-mass clusters at high redshift can provide evidence for an incomplete under-

\footnotetext{
${ }^{30}$ Clay Fellow
}

standing of cosmology, much like the massive clusters at $z>0.3$ discovered in the late 1990s provided evidence against the theoretically preferred $\Omega_{M}=1$ cosmological model of the time (e.g., Carlberg et al. 1996; Henry 1997; Bahcall \& Fan 1998; Donahue et al. 1998).

During the 2009 campaign of the SPT-SZ survey, SPT-CL J2106-5844 was detected with a signal-to-noise ratio $(\mathrm{S} / \mathrm{N})$ of 18.5 in $150 \mathrm{GHz}$ data alone $(\mathrm{S} / \mathrm{N}=22.1$ in multi-band data) - the largest S/N of any cluster in the 2008-2009 SPT-SZ sample. Initial deep optical follow-up observations showed no obvious clustered galaxies in gri, but did show a strong detection of galaxies in the $z$ band. Additional near-infrared and Spitzer photometry further confirmed the presence of clustered galaxies. These extremely red galaxies are consistent with a galaxy cluster at $z>1$.

Chandra observations of SPT-CL J2106-5844 revealed a luminous and extended X-ray source. Optical spectroscopy of member galaxies shows that the cluster is at $z=1.132$. SPT-CL J2106-5844 is therefore not only one of the most massive SPT clusters but also the highest-redshift spectroscopically confirmed SPT cluster. In fact, it is the most massive cluster known at redshift $z>1$, making SPT-CL J2106-5844 an extremely interesting object for detailed study.

We present our initial detection and follow-up observations of SPT-CL J2106-5844 in Section 2. In Section 3, we show that SPT-CL J2106-5844 is a $\gtrsim 10^{15} M_{\odot}$ galaxy cluster at $z=1.132$. We examine the implications of the existence of such a massive cluster at such high redshift in Section 4. We summarize and conclude in Section 5. Throughout this paper, $M_{200}$ and $M_{500}$ masses are defined as the mass enclosed in a spherical region which has a density 200 and 500 times the mean and critical density of the universe, respectively. Uncertainties are reported for $68 \%$ confidence intervals.

\section{OBSERVATIONS, DATA REDUCTION, AND INITIAL FINDINGS}

\subsection{Microwave Observations from the South Pole Telescope}

Currently, the SPT has been used to complete observations of $1500 \mathrm{deg}^{2}$ to full survey depth of $18 \mu \mathrm{K}$ arcmin at $150 \mathrm{GHz}$ and an additional $1000 \mathrm{deg}^{2}$ has been surveyed to a "preview" depth of $54 \mu \mathrm{K}$ arcmin, where the unit $\mathrm{K}$ refers to equivalent fluctuations in the CMB temperature. By the end of the 2011 observing season, we expect the full $2500 \mathrm{deg}^{2}$ survey region to be complete to full depth. Details of the survey strategy and data processing are described in Staniszewski et al. (2009), Vanderlinde et al. (2010), and Williamson et al. (2011). The 2009 observations that yielded the discovery of SPT-CL J21065844 included measurements at 95,150 , and $220 \mathrm{GHz}$ to a noise level of 42,18 , and $85 \mu \mathrm{K}$ arcmin.

SPT-CL J2106-5844 was detected in maps of 2009 SPT data created using time-ordered data processing and map-making procedures equivalent to those described in Vanderlinde et al. (2010). Two different cluster extraction pipelines were used to detect and characterize clusters in these SPT maps. Both pipelines use a multi-scale matched-filter approach similar to the one described in Melin et al. (2006). One pipeline, used to extract clusters from single-band $150 \mathrm{GHz}$ data, is identical to the cluster extraction procedure described in Vanderlinde et al. (2010). The other pipeline, used to extract clusters from multiband SPT data, is identical to that described in Williamson et al. (2011). The final SZ observable produced by both pipelines is $\xi$, the detection significance maximized across all filter scales. 

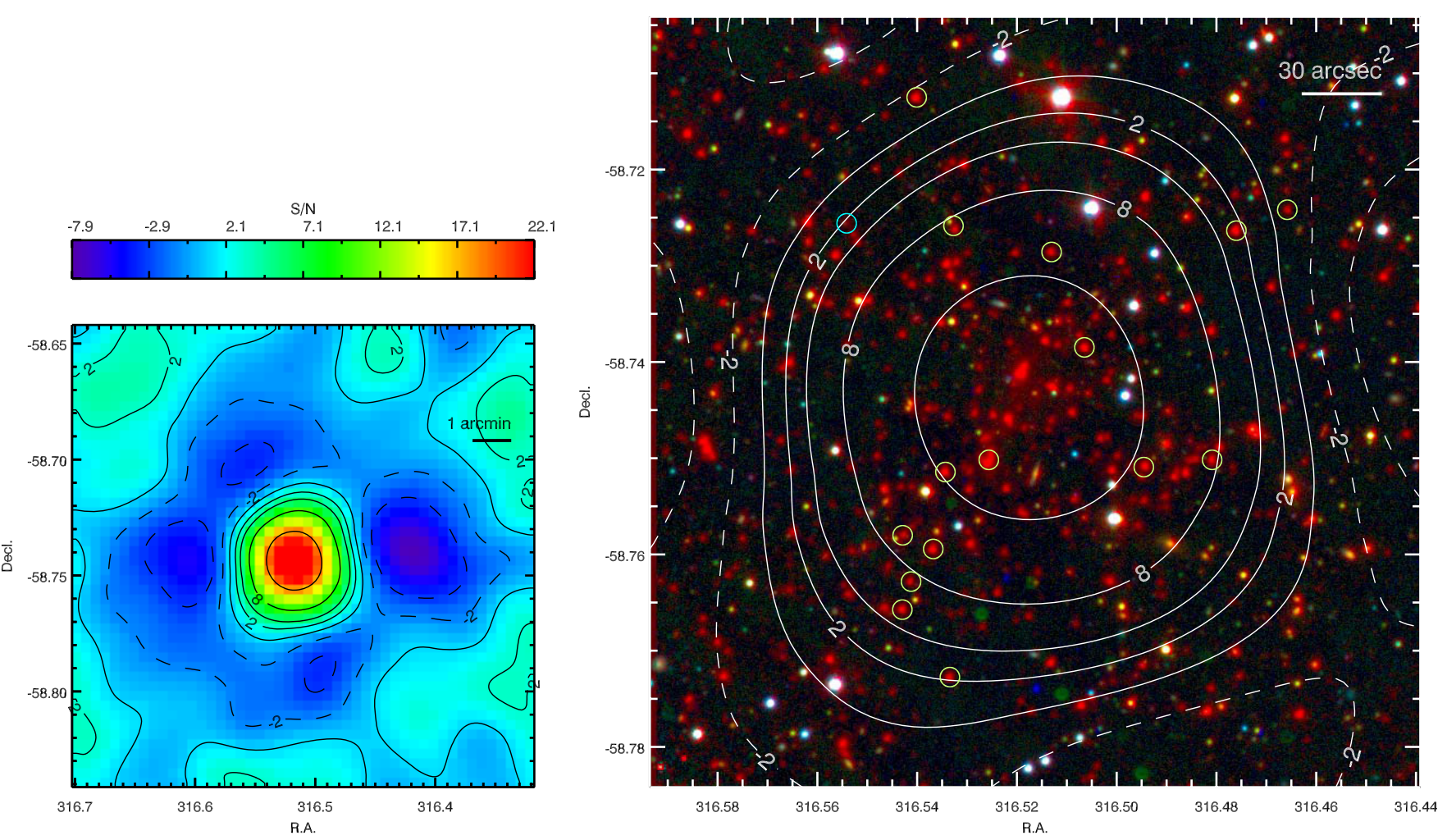

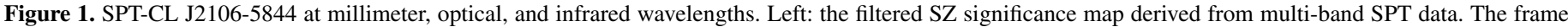

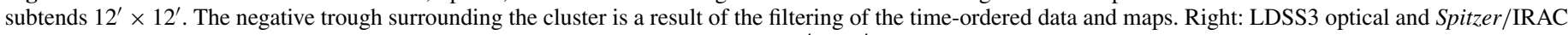

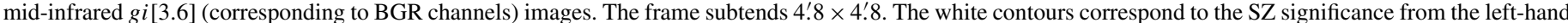

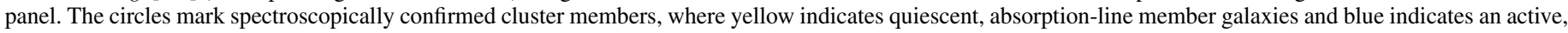
emission-line member galaxy. Some spectroscopic member galaxies are outside the FOV for this image.

(A color version of this figure is available in the online journal.)

SPT-CL J2106-5844 was detected at $\xi=18.5$ by the singleband pipeline and at $\xi=22.1$ by the multi-band pipeline.

An image of the filtered, multi-band SPT data-using the filter that maximized $\xi$, and centered on SPT-CL J2106-5844-is shown in the left panel of Figure 1. Detection significance contours from this same filtered map are overlaid in Figures 1 and 4 .

\subsection{Optical and Infrared Imaging}

In 2009 June, we obtained griz imaging of SPT-CL J21065844 with the Inamori Magellan Areal Camera and Spectrograph (IMACS; Dressler et al. 2006) and Low Dispersion Survey Spectrograph $\left(\operatorname{LDSS}^{31}\right)$ on the twin Magellan $6.5 \mathrm{~m}$ telescopes. We obtained additional $I$-band imaging with FORS2 (Appenzeller et al. 1998) on the Very Large Telescope (VLT) $8 \mathrm{~m}$ telescope in 2010 September. See High et al. (2010) for a description of our observation strategy and reduction procedure.

SPT-CL J2106-5844 was observed at the CTIO $4 \mathrm{~m}$ Blanco telescope using the NEWFIRM imager (Autry et al. 2003) on 2010 July 28. Data were obtained in the $J$ and $K_{s}$ filters under photometric conditions. All images were acquired using a 30 point random dither pattern. At each dither position, six frames with $10 \mathrm{~s}$ exposure times were co-added, providing $60 \mathrm{~s}$ total exposure per position. The dither pattern was repeated as necessary to achieve total exposure times of $7200 \mathrm{~s}$ in $J$ and $4440 \mathrm{~s}$ in $K_{s}$.

NEWFIRM data were reduced using the FATBOY pipeline, originally developed for the FLAMINGOS-2 instrument, and

\footnotetext{
31 http://www.lco.cl/telescopes-information/magellan/instruments-1/ldss-3-1/.
}

modified to work with NEWFIRM data in support of the Infrared Bootes Imaging Survey (A. H. Gonzalez 2011, in preparation). Individual processed frames are combined using SCAMP and SWARP (Bertin et al. 2002), and photometry is calibrated to 2MASS (Skrutskie et al. 2006). The final images in both filters have FWHM of 1".3.

Mid-infrared Spitzer/IRAC imaging was obtained in 2009 September as part of a larger program to follow up clusters identified in the SPT survey. The on-target observations consisted of $8 \times 100 \mathrm{~s}$ and $6 \times 30 \mathrm{~s}$ dithered exposures at 3.6 and $4.5 \mu \mathrm{m}$, respectively. ${ }^{32}$ The deep $3.6 \mu \mathrm{m}$ observations are sensitive to passively evolving cluster galaxies down to $0.1 \mathrm{~L}^{*}$ at $z=1.5$. The data were reduced following the method of Ashby et al. (2009). Briefly, we correct for column pulldown and residual images, mosaic the individual exposures, resample to 0 '.86 pixels (half the solid angle of the native IRAC pixels), and reject cosmic rays.

Images generated from the OIR data are presented in Figure 1. From the deep optical-only images ( $g r i)$, it is difficult to see any indication of a cluster. However, the addition of infrared data shows a clear overabundance of very red galaxies clustered near the peak of the SZ decrement. The overabundance of galaxies that are spatially coincident and have similar colors is a clear indication of a cluster of galaxies. Color-magnitude diagrams (CMDs) for galaxies within the imaging field of view (FOV; Figure 2) show an overabundance of very red galaxies and a probable red sequence. These data are consistent with the spectroscopic redshifts described below.

\footnotetext{
32 In this paper, we will refer to photometry in these bands as [3.6] and [4.5], respectively.
} 


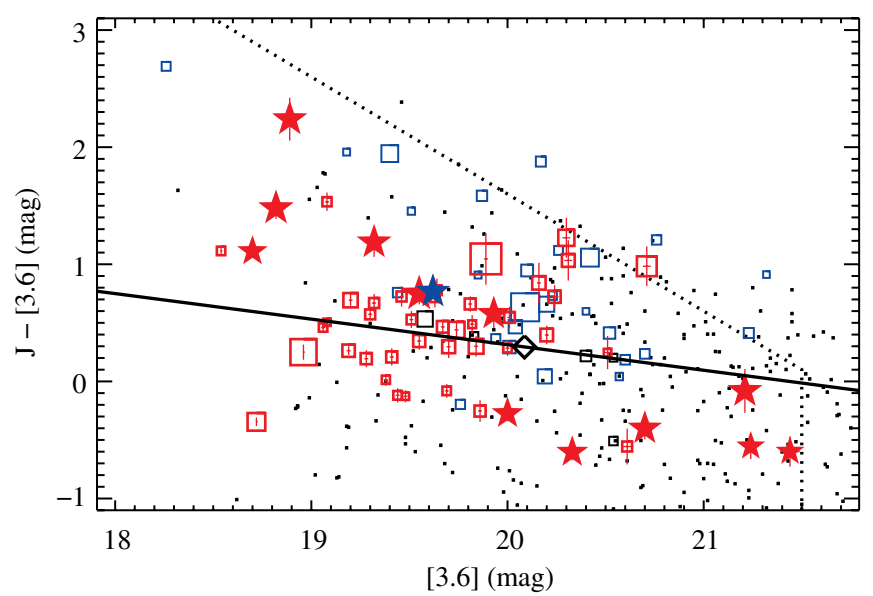

Figure 2. Color-magnitude diagram ( $J-[3.6]$ vs. [3.6]) for galaxies within the IRAC FOV. Suspected red-sequence cluster members are plotted in red. Lower probability, but potential cluster members are plotted in blue. Spectroscopic members are plotted as stars, where the red stars correspond to passive galaxies and the blue star represents an emission-line galaxy. Additional galaxies in the field are plotted as black points. The size of the symbol is inversely proportional to the distance to the center of the cluster as determined by the clustering of the red-sequence galaxies. Our $5 \sigma$ limits are plotted as dotted lines. A red-sequence model corresponding $z=1.132$ is represented as the solid black lines with a representative $L^{*}$ galaxy represented by the black diamond.

(A color version of this figure is available in the online journal.)

\subsection{Optical Spectroscopy}

We obtained low-resolution spectra of several cluster members of SPT-CL J2106-5844 with the Gladders Image-Slicing Multislit Option (GISMO; M. D. Gladders et al. 2011, in preparation) module on IMACS mounted on Magellan in 2010 June; however, these spectra did not yield a definitive cluster redshift. Magellan slit masks were designed using galaxies selected from deep optical and MIR imaging compatible with the red sequence expected at $z \gtrsim 1.2$, as this was the initial redshift approximation from the photometry. The selection was similar to that of Brodwin et al. (2010). This technique gives higher priority to brighter galaxies with colors consistent with the red sequence. If there is a region without a suitable red-sequence target, blue galaxies were selected with the idea that they may be emissionline cluster galaxies. The Magellan observations were obtained using the $f / 2$ camera with the $3001 / \mathrm{mm}$ "red" grism and the WB6300-9500 filter. A total of sixteen $1800 \mathrm{~s}$ exposures were obtained on June 6 and 7. The combination of moderate seeing and a non-optimal filter choice for the actual redshift likely caused the low yield from these observations.

The VLT observations were obtained with the GRIS_300I grism combined with the OG590 blocking filter. Two VLT/ FORS2 masks were designed using galaxies selected from deep I-band VLT pre-imaging and the Spitzer IRAC imaging to have colors compatible with the red sequence expected at $z \approx 1.2-1.3$, which was initially indicated by the photometry. Each mask was populated with approximately 30 slitlets of typical length $10^{\prime \prime}$. This target was originally accepted as Priority B for VLT/FORS2 spectroscopy as part of a larger SPT spectroscopy campaign. In late 2010 November, Chandra $\mathrm{X}$-ray spectroscopy gave an initial redshift from the Fe $\mathrm{K}$ line (see Section 2.4). After this result when it became clear that there was a significant chance of it not being observed in Fall 2010, we submitted a special request to the ESO Director to raise this target to Priority A. That proposal was successful, and on each of three nights in early 2010 December a single $2700 \mathrm{~s}$ exposure was obtained for a total exposure time of $8100 \mathrm{~s}$ on
Table 1

Redshifts for Galaxy Members of SPT-CL J2106-5844

\begin{tabular}{lcccc}
\hline \hline R.A. $\left({ }^{\circ}\right)^{\mathrm{a}}$ & Decl. $\left({ }^{\circ}\right)$ & $z^{\mathrm{b}}$ & Feature $^{\mathrm{c}}$ & Telescope \\
\hline 316.46581 & -58.72418 & $1.1365(6)$ & Ca H\&K & VLT \\
316.47601 & -58.72635 & $1.1347(3)$ & Ca H\&K & VLT \\
316.48048 & -58.69972 & $1.1230(3)$ & [O II] & VLT \\
316.48086 & -58.75017 & $1.1288(2)$ & Ca H\&K & VLT \\
316.49456 & -58.75092 & $1.1148(2)$ & Ca H\&K & VLT \\
$316.50647^{\mathrm{d}}$ & -58.73848 & $1.132(20)$ & D4000 & Magellan \\
$316.50647^{\mathrm{d}}$ & -58.73848 & $1.1296(3)$ & Ca H\&K & VLT \\
316.51307 & -58.72857 & $1.1312(3)$ & Ca H\&K & VLT \\
316.52563 & -58.75017 & $1.1450(6)$ & Ca H\&K & VLT \\
316.53273 & -58.72584 & $1.120(20)$ & D4000 & Magellan \\
316.53346 & -58.77275 & $1.1335(5)$ & Ca H\&K & VLT \\
316.53434 & -58.75143 & $1.1389(4)$ & Ca H\&K & VLT \\
316.53679 & -58.75943 & $1.1274(6)$ & Ca H\&K & VLT \\
316.54011 & -58.71250 & $1.1384(3)$ & Ca H\&K & VLT \\
316.54123 & -58.76282 & $1.1384(5)$ & Ca H\&K & VLT \\
316.54297 & -58.76573 & $1.1260(4)$ & Ca H\&K & Magellan \\
316.54292 & -58.75800 & $1.1363(6)$ & Ca H\&K & VLT \\
316.55414 & -58.72558 & $1.1325(3)$ & [O II] & Magellan \\
316.74972 & -58.72926 & $1.1136(5)$ & Ca H\&K & VLT \\
\hline
\end{tabular}

Notes.

a All coordinates are in $\mathrm{J} 2000$.

${ }^{\mathrm{b}}$ Uncertainties given in units of 0.0001 .

${ }^{c}$ Spectroscopic feature primarily used in determining redshift.

$\mathrm{d}$ This is the same galaxy observed with both Magellan and VLT.

one of the two masks. These observations were taken under excellent seeing conditions $(0.5$ to 0.8$)$ but at high airmass between 1.5 and 1.7. These data were immediately reduced to reveal redshifts of 15 cluster members, enabling a robust cluster redshift.

Spectroscopic reductions proceeded using standard CCD processing with IRAF and the COSMOS reduction package (Kelson 2003) for the VLT and Magellan data, respectively. The data were extracted using the optimal algorithm of Horne (1986). Flux calibration and telluric line removal were performed using the well-exposed continua of spectrophotometric standard stars (Wade \& Horne 1988; Foley et al. 2003).

Three independent redshift determinations were performed using a cross-correlation algorithm (IRAF RVSAO package; Kurtz \& Mink 1998), a template fitting method (SDSS earlytype PCA templates), and a $\chi^{2}$ minimization technique by comparing to galaxy template spectra. There were only minor differences in the final results from the three methods. In total, we have obtained secure redshifts, consistent with membership in a single cluster, for 18 galaxies. Two of these galaxies have obvious [O II] emission, while the others have SEDs consistent with passive galaxies with no signs of ongoing star formation.

A $3 \sigma$ clipping was applied around the peak in redshifts to select spectroscopic cluster members. Representative spectra of cluster members and a redshift histogram of cluster members are presented in Figure 3. Redshift information for cluster members is presented in Table 1. A single galaxy was observed and has a secure redshift from both Magellan and VLT. Although the VLT spectrum shows clear Ca H\&K absorption lines and the Magellan spectrum only shows the D4000 break, the measured redshifts are consistent.

A robust biweight estimator was applied to the spectroscopic sample to determine a mean redshift of $z=1.131_{-0.003}^{+0.002}$ and a velocity dispersion of $\sigma_{v}=1230_{-180}^{+270} \mathrm{~km} \mathrm{~s}^{-1}$. The uncertainty in both quantities is determined through bootstrap resampling. 

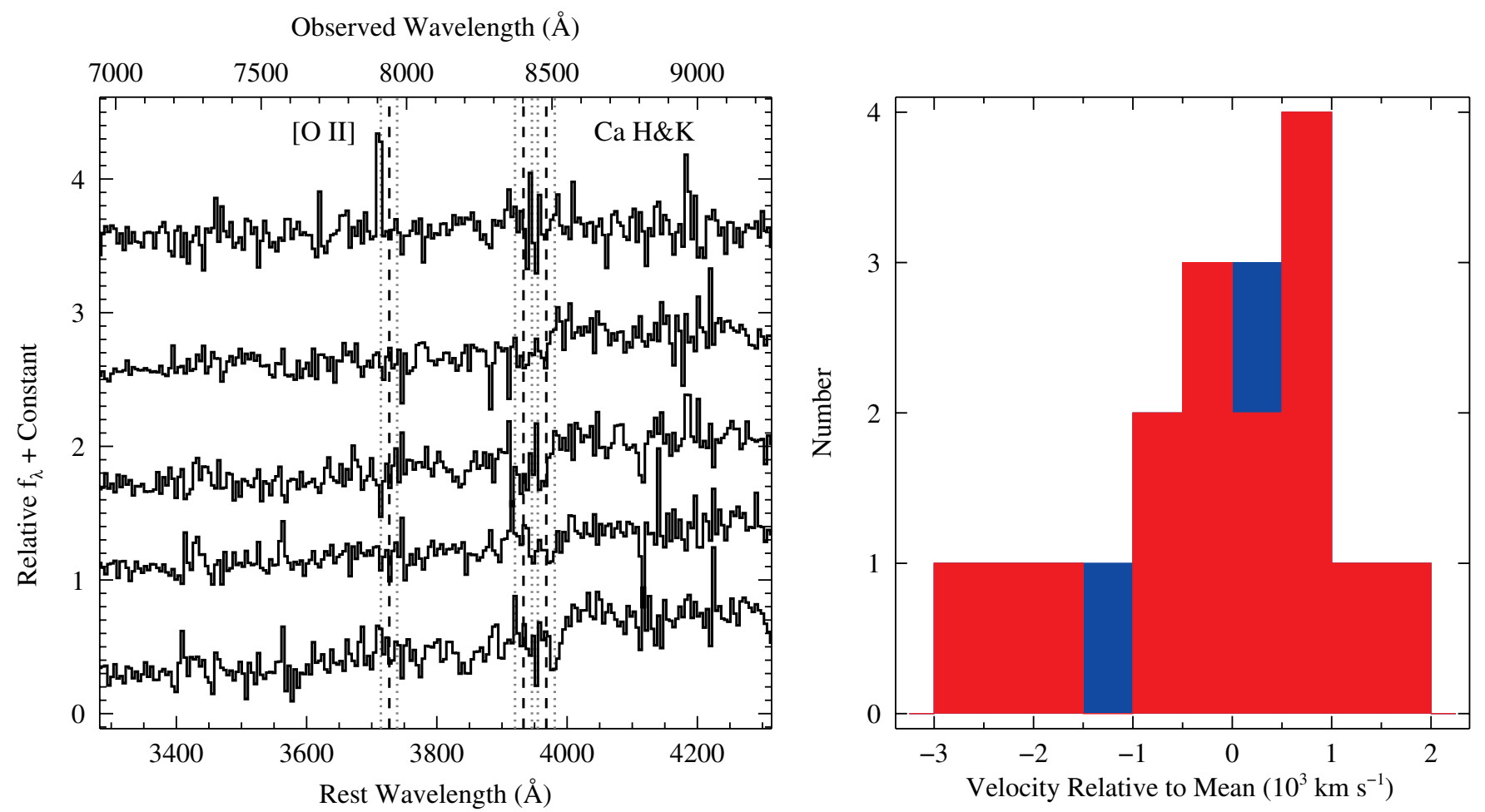

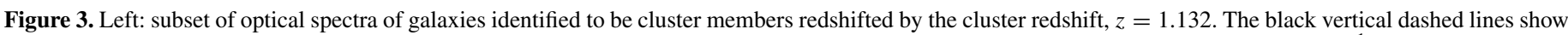

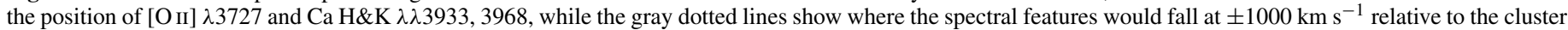

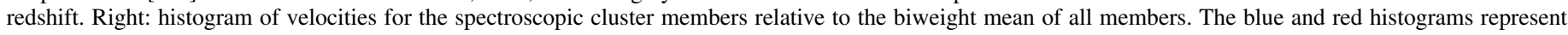
objects with and without obvious [O II] emission, respectively.

(A color version of this figure is available in the online journal.)

Since the dynamics of passive and star-forming galaxies within clusters are different, with passive galaxies assumed to be a better tracer of the cluster potential (e.g., Fadda et al. 1996; Girardi et al. 1996; Mohr et al. 1996; Koranyi \& Geller 2000), we also provide the mean redshift and velocity dispersion of only the passive galaxies, $z=1.132_{-0.003}^{+0.002}$ and $\sigma_{v}=1270_{-210}^{+320} \mathrm{~km} \mathrm{~s}^{-1}$, respectively.

The velocity distribution of spectroscopic members relative to the mean velocity (Figure 3) is skewed. The bootstrap resampling produced a positive skewness in $89 \%$ of the samples, which indicates that it is not very statistically significant given our sample size. However, this may be an indication of substructure, consistent with the possible merger seen in the $\mathrm{X}$-ray image (see Section 2.4). There is no spatial segregation of galaxies clustered in velocity space; however, the number of spectroscopic members is still small.

\subsection{X-ray Observations}

As part of a program to follow up the most massive, high redshift clusters detected with the SPT in 2009, we have obtained a 25 ks X-ray observation of SPT-CL J2106-5844. These data were obtained through a combined Chandra High Resolution Camera and Advanced CCD Imaging Spectrometer Guaranteed Time Observation program. Data reduction was performed with the method described by Andersson et al. (2010). The observation resulted in 1425 source counts within $r_{500}$ in the $0.5-7.0 \mathrm{keV}$ band, corresponding to a flux, $F_{X}(0.5-2.0 \mathrm{keV})=$ $(2.58 \pm 0.15) \times 10^{-13} \mathrm{erg} \mathrm{cm}^{-2} \mathrm{~s}^{-1}$. A $2^{\prime \prime} \times 2^{\prime \prime}$-binned X-ray image was produced using the X-ray events in the $0.7-4.0 \mathrm{keV}$ band (Figure 4). The observation reveals an X-ray luminous cluster with pronounced asymmetry in the surface brightness distribution (see Figure 4), indicating that it may be in a merging state. In particular, we note that a bright concentration of gas is offset $\sim 10^{\prime \prime}$ east of the main cluster centroid.

X-ray observations of SPT-CL J2106-5844 were used to measure the gas temperature, $T_{X}$, and the X-ray analog of Comptonization, $Y_{X}=M_{\text {gas }} \times T_{X}$. The cluster mass can then be inferred with the $M-Y_{X}$ and $M-T_{X}$ relations of Vikhlinin et al. (2009). The $r_{500}$ radius is derived iteratively from the $M-Y_{X}$ scaling relation as described in Andersson et al. (2010). See Section 3.3 for details regarding the X-ray mass estimates. A spectrum is extracted from the data within the derived $r_{500}$ radius, excluding the core within $0.15 r_{500}$. The spectrum is fit, keeping the absorbing hydrogen column fixed at the galactic value $n_{H}=4.32 \times 10^{20} \mathrm{~cm}^{-2}$, and the metal abundance fixed at 0.3 solar. Fixing the redshift to the mean spectroscopic redshift, $z=1.132$, results in a best-fit $T_{X}=11.0_{-1.9}^{+2.6} \mathrm{keV}$ and $Y_{X}=(14.3 \pm 3.4) \times 10^{14} M_{\odot} \mathrm{keV}$. Within $r_{500}\left(117^{\prime \prime}\right)$, including the cluster core, SPT-CL J2106-5844 has a luminosity of $L_{X}(0.5-2.0 \mathrm{keV})=(13.9 \pm 1.0) \times 10^{44} \mathrm{erg} \mathrm{s}^{-1}$, comparable to the luminosity of the Bullet cluster (Markevitch et al. 2002).

An X-ray spectrum was produced from the data within $20^{\prime \prime}$ $\left(0.17 r_{500}\right)$, where the metal abundance of the gas is likely to peak. The resulting spectrum is shown in Figure 5 with a model folded through the detector response. The strong $6.7 \mathrm{keV}$ Fe $\mathrm{K}$ line is present in the spectrum at approximately $3 \mathrm{keV}$. This spectrum indicates $z=1.18 \pm 0.03$, a metal abundance $Z=1.44_{-0.51}^{+0.66} Z_{\odot}$, and a central temperature $T_{X}=6.5_{-1.1}^{+1.7} \mathrm{keV}$. Notably, this measurement was the first robust spectroscopic redshift measurement for this cluster.

Merger simulations suggest that high-redshift cool-core clusters should be rare (Gottlöber et al. 2001), and previous 

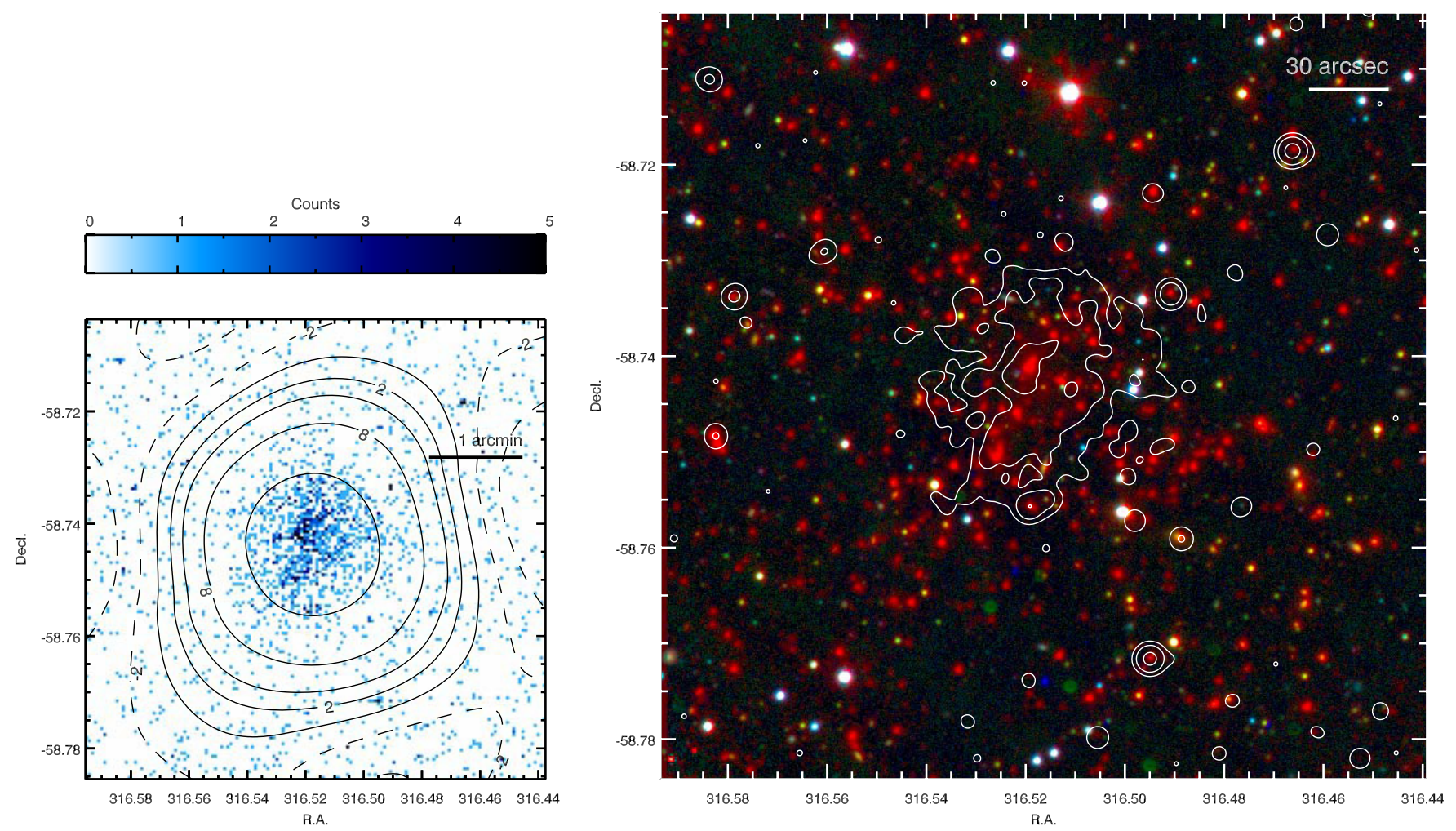

Figure 4. SPT-CL J2106-5844 at X-ray, millimeter, optical, and infrared wavelengths. Left: Chandra X-ray image from the $0.7-4.0 \mathrm{keV}$ band, with $4 \times 4$ binning of the original ACIS pixels ( 0 '.49 on a side). The black contours correspond to the SZ significance from the left-hand panel of Figure 1. Right: LDSS3 optical and Spitzer/IRAC mid-infrared gi [3.6] (corresponding to BRG channels) images. The frame subtends $4.8 \times 4.8$. The white contours are the smoothed X-ray signal from the left panel.

(A color version of this figure is available in the online journal.)
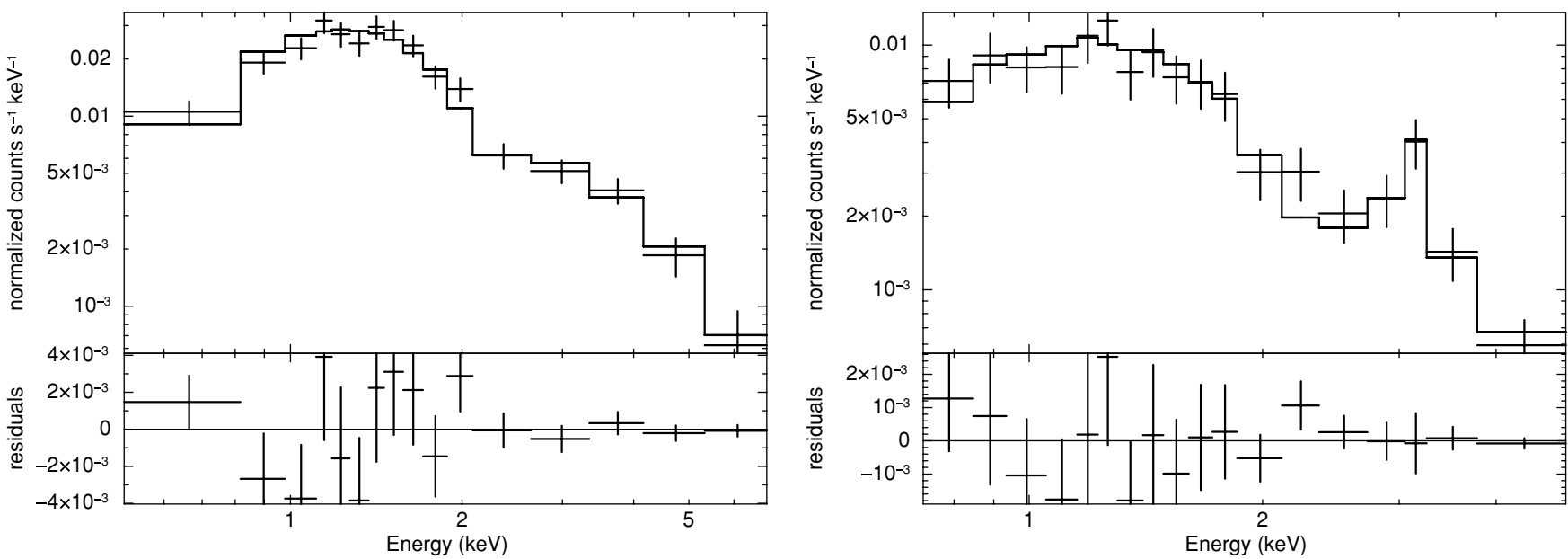

Figure 5. Core-excluded (circular annulus spanning 0.15-1 $r_{500}$ ) and central region (circular aperture with radius $0.17 r_{500}$ ) X-ray spectra of SPT-CL J2106-5844 (left and right panels, respectively). Overplotted (solid line) is a fit APEC spectrum redshifted to $z=1.18$ and folded through the detector sensitivity. The $6.7 \mathrm{keV}$ Fe $\mathrm{K}$ line observed at $\sim 3 \mathrm{keV}$ is both obvious and strong for the spectrum from the central region.

observations have indicated a steep decline in the number of cool-core clusters at high redshift (e.g., Vikhlinin et al. 2007). However, other analyses have found that the cool-core fraction varies significantly between different high redshift X-ray selected samples (e.g., Santos et al. 2010). Future work comparing these results to the cool-core fraction in SZ selected samples should help to understand the role of selection in these results. Regardless of the abundance at high redshift, SPT-CL J21065844 is one of the few high-redshift cool-core galaxy clusters known.

\section{MASS ESTIMATES FROM CLUSTER OBSERVABLES}

In estimating the mass of a galaxy cluster from any observable property, care must be taken to properly account for Eddington bias, which, in this particular case, refers to the preferential scattering of lower-mass systems into a given observable bin due to a steeply falling source population. This is handled through application of Bayes' theorem: we use scaling relations and noise measurements to calculate conditional probabilities of observable $O$ at fixed mass, $P(O \mid M)$, and combine these 
with a mass prior $P(M)$ to yield a posterior estimate of the mass $P(M \mid O)$. When the observable $O$ is the same quantity that was used to detect the cluster, the prior probability of the cluster's mass is simply the cosmological mass function. However, when the observable in question is from a targeted follow-up observation, the prior must also take into account the selection function of the survey in which it was discovered. For follow-up observations targeting a single, very rare or interesting object, such as SPT-CL J2106-5844, the appropriate prior mass distribution is strongly influenced by the selection. For this reason, we report masses derived from follow-up observations with a flat mass prior only. We report both flat-prior and massfunction-prior masses for the SZ-derived mass. In Section 3.4, we combine the conditional probabilities derived from different observables and apply the mass-function prior to the result in order to determine the best mass estimate based on all the available information. For the cosmological analyses in Section 4 , we use only this joint posterior mass probability density.

\subsection{SZ Mass Estimate}

An SZ-based cluster mass estimate is produced following Vanderlinde et al. (2010), using the single-band $(150 \mathrm{GHz})$ detection significance $\xi$ as a mass proxy. We direct the reader to that work for details. Briefly, an SZ-mass scaling relation, derived from the simulations of Shaw et al. (2009) and constrained though a cosmological analysis, is used to estimate a set of conditional probabilities $P(\xi \mid M)$, taking into account the measurement noise and the intrinsic scatter in the scaling relation. A posterior mass estimate is then calculated through application of a mass prior. The systematic errors are estimated by linearly expanding the mass estimate as a function of the scaling relation parameters and using the marginalized uncertainties on these parameters.

The SZ detection of this cluster is from an untargeted survey, allowing us to take the halo mass function of Tinker et al. (2008) as a prior on its mass. We assume the WMAP 7-year preferred $\Lambda$ CDM parameters (Larson et al. 2011) when calculating this prior; marginalizing over the $\Lambda \mathrm{CDM}$ cosmological parameter uncertainties was found to have a negligible impact on the effect of this prior. SPT-CL J2106-5844 has a single-band $150 \mathrm{GHz}$ significance of $\xi=18.5$, which results in an unbiased posterior SZ-derived mass estimate of $M_{\mathrm{SZ}, 200}=$ $(1.06 \pm 0.23) \times 10^{15} h_{70}^{-1} M_{\odot}$.

We also report (see Section 3.4 and Table 2) a biased SZ mass estimate, using a flat mass prior; we present it here for completeness only. It is important to note that this mass is not directly comparable to other flat-mass-prior estimates (e.g., the masses estimated in Sections 3.2 and 3.3). The SZ observation was drawn from an untargeted survey and hence from the entire underlying population of clusters (given by the halo mass function), while the targeted observations were conducted with a priori knowledge that a massive cluster was present; these two different modes of observation draw from different populations and thus will exhibit different levels of Eddington bias. To reiterate, naively using any mass estimate without understanding the selection of the observed population can result in biased measurements.

\subsection{Dynamical Mass Estimate}

The dynamical mass of SPT-CL J2106-5844 can be estimated from the velocity dispersion using the $M-\sigma_{v}$ calibration
Table 2

Mass Estimates for SPT-CL J2106-5844

\begin{tabular}{lcc}
\hline \hline Observable & Measurement & $M_{200}\left(10^{15} h_{70}^{-1} M_{\odot}\right)$ \\
\hline SZ $\xi$ & 18.5 & $\mathbf{1 . 0 6} \pm \mathbf{0 . 2 3}$ \\
SZ $\xi$ (flat prior) & 18.5 & $1.24 \pm 0.30$ \\
$Y_{X}$ & $(14.3 \pm 3.4) \times 10^{14} M_{\odot} \mathrm{keV}$ & $1.85 \pm 0.40$ \\
$T_{X}$ & $11.0_{-1.9}^{+2.6} \mathrm{keV}^{+310} \mathrm{~km} \mathrm{~s}^{-1}$ & $1.83 \pm 0.76$ \\
$\sigma_{v}{ }^{\mathrm{a}}$ & $1270_{-220}^{+3} \mathrm{u}^{-1.7}$ \\
\hline Combined & $\ldots$ & $\mathbf{1 . 2 7} \pm \mathbf{0 . 2 1}$
\end{tabular}

Notes. Unbolded masses indicate Eddington-biased mass estimates, calculated using flat priors on mass. Note that the SZ (untargeted) measurement suffers from a considerably different Eddington bias than the other (targeted) flat-prior estimates. The probability distribution for the combined mass was obtained by multiplying the unbiased (bold) SZ mass probability by the flat-prior $Y_{X}$ mass probability (see the text for details). Only the unbiased SZ $\xi$ and $Y_{X}$ mass estimates were used to generate the combined mass estimate.

a As determined by the biweight estimate of the passive galaxies.

of Evrard et al. (2008). Numerical simulations indicate that galaxies are likely to exhibit velocity bias compared to the dark matter velocity field (e.g., Biviano et al. 2006; White et al. 2010). Mock observations of simulated clusters that model the galaxy selection within follow-up observations like those reported here enable these biases to be quantified and corrected (A. Saro et al. 2011, in preparation). Published results suggest these biases are at the level of $10 \%$ in the dispersion (White et al. 2010). Here, we attempt no correction to the dispersion but account for this additional systematic uncertainty in the mass estimator.

As the dynamical mass estimate arises from a targeted followup observation, the underlying mass distribution can be well defined (and accounted for) only in the context of the SZ-based selection. Such a joint estimate of mass is presented below in Section 3.4; here, we report an independent (hence biased) dynamical mass estimate using a flat prior on the mass.

Using the velocity dispersion from all 18 spectroscopic cluster members gives $M_{\text {dyn,200 }}=1.3_{-0.5}^{+1.4} \times 10^{15} h_{70}^{-1} M_{\odot}$. Using the velocity dispersion from only the passive galaxies provides a very similar result, $\left.M_{\text {dyn, } 200}=1.4_{-0.8}^{+1.7} \times 10^{15} h_{70}^{-1} M_{\odot}\right)$. In both cases, the uncertainty takes into account the statistical uncertainty of the velocity dispersion, an additional $13 \%$ intrinsic scatter of the velocity dispersion due to the orientation of the velocity ellipsoid along the line of sight (White et al. 2010), the 4\% intrinsic scatter of the Evrard et al. (2008) $M-\sigma_{v}$ relation, and a $10 \%$ estimate of the systematic uncertainty in the dispersion that arises from the currently uncertain kinematic relationship between the galaxies and the underlying dark matter.

\subsection{X-ray Mass Estimate}

As described in Section 2.4, the X-ray observations of SPT-CL J2106-5844 were used to measure the gas temperature, $T_{X}$, and the X-ray analog of Comptonization, $Y_{X}=M_{\text {gas }} \times T_{X}$. Following the methodology of Andersson et al. (2010), the data were iteratively fit to determine $r_{500}, T_{X}$, and $Y_{X}$. Using the scaling relations of Vikhlinin et al. (2009), these values provide estimates of the gas and total mass of the cluster, $M_{\text {gas, } 500}=(1.27 \pm 0.07) \times 10^{14} h_{70}^{-1} M_{\odot}$ and $M_{Y_{X}, 500}=$ $(0.93 \pm 0.19) \times 10^{15} h_{70}^{-1} M_{\odot}$, respectively. Estimating the cluster mass from the gas temperature, we find a similarly high mass of $M_{T_{X}, 500}=(0.92 \pm 0.37) \times 10^{15} h_{70}^{-1} M_{\odot}$, where we have increased the mass by $17 \%$ according to the recipe in Vikhlinin 
et al. (2009) because of the large degree of asymmetry in the surface brightness distribution, indicative of merging activity.

For both mass estimates above, we have adopted a systematic uncertainty of $9 \%$ for the mass calibration of the scaling relations. The uncertainty on the self-similar evolution of these relations was estimated using the simulation informed estimate of $5 \%$ and $7 \%$ uncertainty on the normalization at $z=0.6$ for the $M-Y_{X}$ and $M-T_{X}$, respectively. This uncertainty on the evolutionary term was extrapolated to the cluster redshift. For further discussion on these uncertainties, see Vikhlinin et al. (2009); the appropriate mass prior to account for Eddington bias is only well-defined in the context of SZ-based selection.

\subsection{Combined Mass Estimate}

The X-ray, SZ, and optical redshift data can each be used to produce an estimate of the mass of SPT-CL J2106-5844. We list our estimates for the mass of SPT-CL J2106-5844 from each of these observables in Table 2. Note that we have converted the $M_{Y_{X}, 500}$ mass to $M_{Y_{X}}, 200$ assuming a Navarro-Frenk-White density profile and the mass-concentration relation of Duffy et al. (2008). $M_{T_{X}, 500}$ is not used in the combined mass estimate since it is correlated with $M_{Y_{X}}, 500$ and has a larger uncertainty. Similarly, $M_{\mathrm{dyn}, 200}$ is not used in the combined mass estimate because of its large uncertainty.

We assume for the purposes of the combined mass estimate that the uncertainties are uncorrelated between the two masses. In principle, the different observables could have correlated scatter in their scaling with mass; however, this is expected to be negligible at the current $\mathrm{S} / \mathrm{N}$ of the observables. This simplification allows us to write the posterior mass distribution as a product of the two independent conditional probabilities,

$$
P\left(M \mid \xi, Y_{X}\right) \propto P(M) \cdot P(\xi \mid M) \cdot P\left(Y_{X} \mid M\right),
$$

where $P(M)$ is again the halo mass function of Tinker et al. (2008), evaluated at the WMAP 7-year preferred point in $\Lambda$ CDM parameter space. Functionally, this reduces to a product of two probability density functions, the unbiased SZ mass estimate and the flat-prior $Y_{X}$ mass estimate. We find the combined, unbiased, mass estimate from the two observables to be $M_{200}=$ $(1.27 \pm 0.21) \times 10^{15} h_{70}^{-1} M_{\odot}$.

All mass estimates are consistent and very high for a cluster at this redshift. Each individual mass estimate, as well as the combined estimate, indicates that SPT-CL J2106-5844 is the most massive cluster yet discovered at $z>1$. It is $\sim 60 \%$ more massive than SPT-CL J0546-5345 (Brodwin et al. 2010) at $z=1.07$, the second most massive galaxy cluster at $z>1$.

\section{LIKELIHOOD ANALYSIS}

Since SPT-CL J2106-5844 is the highest S/N cluster found in 2008-2009 SPT survey fields, the highest-redshift spectroscopically confirmed SPT cluster, and the most massive cluster discovered at $z>1$ by any method, it raises the question: Is the existence of SPT-CL J2106-5844 consistent with a Gaussiandistributed primordial density field in a $\Lambda$ CDM universe?

We investigate the probability of finding a cluster with the mass and redshift of SPT-CL J2106-5844 in the context of $\Lambda \mathrm{CDM}$ as follows. Based on the likelihood calculation of Vanderlinde et al. (2010) and the CosmoMC software package (Lewis \& Bridle 2002), we produce an MCMC chain sampling the parameter space of spatially flat $\Lambda \mathrm{CDM}$ cosmology, storing the halo mass function of Tinker et al. (2008) at each step in the chain. Current data sets, consisting of the WMAP 7-year data (Larson et al. 2011) and the SPT 2008 cluster catalog (Vanderlinde et al. 2010), are used to constrain the parameter space explored. This yields a set of halo mass functions representative of current uncertainties of $\Lambda \mathrm{CDM}$ parameter space.

Integrating each of these mass functions, we compute the Poisson likelihood of finding a cluster at or above the mass and redshift of SPT-CL J2106-5844 in a $2500 \mathrm{deg}^{2}$ field. $^{33}$ For the MCMC chain described above, the existence of a cluster at or above the mass and redshift of SPT-CL J21065844 is less than $5 \%$ likely in models contributing $32 \%$ of the WMAP7+V10 probability. Marginalized over all WMAP7+V10 allowed parameter space, we find that there is a $7 \%$ chance of finding a cluster at least as massive and at a redshift at least as high as SPT-CL J2106-5844 in the SPT survey field. Prior to having observed this cluster, the expectation would be that there is only one galaxy cluster at least as massive and at a redshift at least as high as SPT-CL J2106-5844 in the entire sky.

\section{CONCLUSIONS}

We present the discovery and initial characterization of SPT-CL J2106-5844. This massive cluster was discovered in the $2500 \mathrm{deg}^{2}$ SPT survey as a highly significant SZ decrement. Initial follow-up in the optical and infrared indicated that it was both at high redshift and very rich. Optical spectroscopy yielded a cluster redshift of $z=1.132$, consistent with the redshift found from the X-ray spectrum of the hot intracluster gas, $z=1.18$, and the broad-band SEDs of member galaxies. SPT-CL J2106-5844 is currently the highest-redshift SZdiscovered galaxy cluster.

SPT-CL J2106-5844 is also extremely massive. X-ray, SZ, and velocity dispersion data provide complementary and consistent measurements of the mass (see Table 2). The best combined estimate of the mass is $M_{200}=(1.27 \pm 0.21) \times 10^{15} h_{70}^{-1} M_{\odot}$, making SPT-CL J2106-5844 the most massive galaxy cluster yet discovered at $z>1$. The combination of its high mass and high redshift make SPT-CL J2106-5844 a very interesting object in which to study galaxy formation and evolution only a few Gyr after the formation of the first stars. The X-ray data show that SPT-CL J2106-5844 has a cool core and may be currently undergoing a merger. The distribution of galaxy velocities is slightly skewed, but is consistent with a Gaussian distribution. Additional spectroscopy may help determine if there is significant substructure in the cluster.

The high mass and redshift of SPT-CL J2106-5844 also make it an interesting object from a cosmological perspective. The chances of finding a cluster this extreme in mass and redshift in a $2500 \mathrm{deg}^{2}$ survey is only $7 \%$, given current cosmological parameter constraints, with only one such cluster expected in the entire sky. As the SPT cluster catalog grows, and our understanding of the various mass-observable scaling relations improves, SZ-discovered clusters will enable yet more powerful tests of the current cosmological paradigm.

The South Pole Telescope program is supported by the National Science Foundation through grant ANT-0638937. Partial support is also provided by the NSF Physics Frontier

\footnotetext{
33 Although SPT-CL J2106-5844 was found in the $1500 \mathrm{deg}^{2}$ region that has been observed to full survey depth, any galaxy cluster that is more massive than SPT-CL J2106-5844 should have been detected in the shallower observations obtained for the additional $1000 \mathrm{deg}^{2}$ observed with SPT (Williamson et al. 2011).
} 
Center grant PHY-0114422 to the Kavli Institute of Cosmological Physics at the University of Chicago, the Kavli Foundation, and the Gordon and Betty Moore Foundation. This work is based in part on observations obtained with the Spitzer Space Telescope (PID 60099), which is operated by the Jet Propulsion Laboratory, California Institute of Technology under a contract with NASA. Support for this work was provided by NASA through an award issued by JPL/Caltech. Additional data were obtained with the $6.5 \mathrm{~m}$ Magellan Telescopes located at the Las Campanas Observatory, Chile. Support for X-ray analysis was provided by NASA through Chandra Project Numbers 12800071 and 12800088 issued by the Chandra X-ray Observatory Center, which is operated by the Smithsonian Astrophysical Observatory for and on behalf of NASA under contract NAS8-03060. Observations from VLT programs 086.A-0741 and 286.A-5021 were included in this work. We acknowledge the use of the Legacy Archive for Microwave Background Data Analysis (LAMBDA). Support for LAMBDA is provided by the NASA Office of Space Science. Galaxy cluster research at Harvard is supported by NSF grant AST-1009012. Galaxy cluster research at SAO is supported in part by NSF grants AST1009649 and MRI-0723073. The McGill group acknowledges funding from the National Sciences and Engineering Research Council of Canada, Canada Research Chairs program, and the Canadian Institute for Advanced Research. X-ray research at the CfA is supported through NASA Contract NAS 8-03060. The Munich group acknowledges support from the Excellence Cluster Universe and the DFG research program TR33. R.J.F. is supported by a Clay Fellowship. B.A.B. is supported by a KICP Fellowship, support for M. Brodwin was provided by the W. M. Keck Foundation, M. Bautz acknowledges support from contract 2834-MIT-SAO-4018 from the Pennsylvania State University to the Massachusetts Institute of Technology. A.C. acknowledges the support of grants Basal CATA PFB 06/09 and FONDAP No. 15010003. M.D. acknowledges support from an Alfred P. Sloan Research Fellowship, W.F. and C.J. acknowledge support from the Smithsonian Institution, and B.S. acknowledges support from the Brinson Foundation.

Facilities: Blanco (NEWFIRM), CXO (ACIS), Magellan: Baade (IMACS), Magellan:Clay (LDSS3), Spitzer (IRAC), SPT, VLT:Antu (FORS2)

\section{REFERENCES}

Andersson, K., et al. 2010, arXiv:1006.3068 Appenzeller, I., et al. 1998, Messenger, 94, 1 Ashby, M. L. N., et al. 2009, ApJ, 701, 428

Autry, R. G., et al. 2003, Proc. SPIE, 4841, 525

Bahcall, N. A., \& Fan, X. 1998, ApJ, 504, 1

Bertin, E., Mellier, Y., Radovich, M., Missonnier, G., Didelon, P., \& Morin, B. 2002, in ASP Conf. Ser. 281, Astronomical Data Analysis Software and Systems XI, ed. D. A. Bohlender, D. Durand, \& T. H. Handley (San Francisco, CA: ASP), 228

Biviano, A., Murante, G., Borgani, S., Diaferio, A., Dolag, K., \& Girardi, M. 2006, A\&A, 456, 23

Brodwin, M., et al. 2006, ApJ, 651, 791
Brodwin, M., et al. 2010, ApJ, 721, 90

Carlberg, R. G., Yee, H. K. C., Ellingson, E., Abraham, R., Gravel, P., Morris, S., \& Pritchet, C. J. 1996, ApJ, 462, 32

Carlstrom, J. E., Holder, G. P., \& Reese, E. D. 2002, ARA\&A, 40, 643

Carlstrom, J. E., et al. 2009, arXiv:0907.4445

Colafrancesco, S., Lucchin, F., \& Matarrese, S. 1989, ApJ, 345, 3

De Lucia, G., \& Blaizot, J. 2007, MNRAS, 375, 2

Donahue, M., Voit, G. M., Gioia, I., Lupino, G., Hughes, J. P., \& Stocke, J. T. 1998, ApJ, 502, 550

Dressler, A., Hare, T., Bigelow, B. C., \& Osip, D. J. 2006, Proc. SPIE, 6269, $62690 \mathrm{~F}$

Duffy, A. R., Schaye, J., Kay, S. T., \& Dalla Vecchia, C. 2008, MNRAS, 390, L64

Eisenhardt, P. R. M., et al. 2008, ApJ, 684, 905

Evrard, A. E., et al. 2008, ApJ, 672, 122

Fadda, D., Girardi, M., Giuricin, G., Mardirossian, F., \& Mezzetti, M. 1996, ApJ, 473,670

Foley, R. J., et al. 2003, PASP, 115, 1220

Girardi, M., Fadda, D., Giuricin, G., Mardirossian, F., Mezzetti, M., \& Biviano, A. $1996, \mathrm{ApJ}, 457,61$

Gottlöber, S., Klypin, A., \& Kravtsov, A. V. 2001, ApJ, 546, 223

Henry, J. P. 1997, ApJ, 489, L1

High, F. W., et al. 2010, ApJ, 723, 1736

Horne, K. 1986, PASP, 98, 609

Kelson, D. D. 2003, PASP, 115, 688

Koranyi, D. M., \& Geller, M. J. 2000, AJ, 119, 44

Kurtz, M. J., \& Mink, D. J. 1998, PASP, 110, 934

Larson, D., et al. 2011, ApJS, 192, 16

Lewis, A., \& Bridle, S. 2002, Phys. Rev. D, 66, 103511

Lucchin, F., \& Matarrese, S. 1988, ApJ, 330, 535

Markevitch, M., Gonzalez, A. H., David, L., Vikhlinin, A., Murray, S., Forman, W., Jones, C., \& Tucker, W. 2002, ApJ, 567, L27

Marriage, T. A., et al. 2010, arXiv:1010.1065

Melin, J.-B., Bartlett, J. G., \& Delabrouille, J. 2006, A\&A, 459, 341

Mohr, J. J., Geller, M. J., Fabricant, D. G., Wegner, G., Thorstensen, J., \& Richstone, D. O. 1996, ApJ, 470, 724

Mortonson, M. J., Hu, W., \& Huterer, D. 2011, Phys. Rev. D, 83, 023015

Mullis, C. R., Rosati, P., Lamer, G., Böhringer, H., Schwope, A., Schuecker, P., \& Fassbender, R. 2005, ApJ, 623, L85

Muzzin, A., et al. 2009, ApJ, 698, 1934

Papovich, C., et al. 2010, ApJ, 716, 1503

Rosati, P., et al. 2004, AJ, 127, 230

Rosati, P., et al. 2009, A\&A, 508, 583

Santos, J. S., Tozzi, P., Rosati, P., \& Böhringer, H. 2010, A\&A, 521, A64

Shaw, L. D., Zahn, O., Holder, G. P., \& Doré, O. 2009, ApJ, 702, 368

Skrutskie, M. F., et al. 2006, AJ, 131, 1163

Stanford, S. A., et al. 2005, ApJ, 634, L129

Stanford, S. A., et al. 2006, ApJ, 646, L13

Staniszewski, Z., et al. 2009, ApJ, 701, 32

Sunyaev, R. A., \& Zeldovich, Y. B. 1972, Comments Astrophys. Space Phys., 4, 173

Tinker, J., Kravtsov, A. V., Klypin, A., Abazajian, K., Warren, M., Yepes, G., Gottlöber, S., \& Holz, D. E. 2008, ApJ, 688, 709

Vanderlinde, K., et al. 2010, ApJ, 722, 1180

Verde, L., Jimenez, R., Kamionkowski, M., \& Matarrese, S. 2001, MNRAS, 325,412

Vikhlinin, A., Burenin, R., Forman, W. R., Jones, C., Hornstrup, A., Murray, S. S., \& Quintana, H. 2007, in Heating versus Cooling in Galaxies and Clusters of Galaxies, ed. H. Böhringer, G. W. Pratt, A. Finoguenov, \& P. Schuecker (Berlin: Springer), 48

Vikhlinin, A., et al. 2009, ApJ, 692, 1060

Wade, R. A., \& Horne, K. 1988, ApJ, 324, 411

White, M., Cohn, J. D., \& Smit, R. 2010, MNRAS, 408, 1818

Williamson, R., et al. 2011, ApJ, submitted (arXiv:1101.1290)

Wilson, G., et al. 2009, ApJ, 698, 1943 